\title{
A missense variant rs2585405 in clock gene PER1 is associated with the increased risk of noise-induced hearing loss in a Chinese occupational population
}

Hao Chen ${ }^{1 \dagger}$, Xuexue Ding ${ }^{1 \dagger}$, Enmin Ding ${ }^{2 \dagger}$, Mengyao Chen ${ }^{1}$, Huimin Wang ${ }^{3}$, Guangzhi Yang ${ }^{3}$ and Baoli Zhu ${ }^{1,2^{*}}$

\begin{abstract}
Objective: To investigate the potential association of cochlear clock genes (CRY1, CRY2, PER1, and PER2), the DNF gene (brain-derived neurotrophic factor), and the NTF3 gene (neurotrophin3) with susceptivity to noise-induced hearing loss (NIHL) among Chinese noise-exposed workers.

Methods: A nested case-control study was performed with 2056 noise-exposed workers from a chemical fiber factory and an energy company who underwent occupational health examinations in 2019 as study subjects. Propensity score matching was conducted to screen cases and controls by matching sex, age, and the consumption of tobacco and alcohol. A total of 1269 participants were enrolled. Then, general information and noise exposure of the study subjects were obtained through a questionnaire survey and on-site noise detection. According to the results of audiological evaluations, the participants were divided into the case group $(n=432$, high-frequency threshold shift $>25 d B)$ and the matched control group ( $n=837$, high-frequency threshold shift $\leq 25 \mathrm{~dB}$ ) by propensity score matching. Genotyping for PER1 rs2253820 and rs2585405; PER2 rs56386336 and rs934945; CRY1 rs 1056560 and rs3809236; CRY2 rs2292910 and rs6798; BDNF rs1 1030099, rs7124442 and rs6265; and NTF3 rs 1805149 was conducted using the TaqMan-PCR technique.
\end{abstract}

Results: In the dominant model and the co-dominant model, the distribution of PER 1 rs 2585405 genotypes between the case group and the control group was significantly different $(P=0.03, P=0.01)$. The NIHL risk of the subjects with the GC genotype was 1.41 times the risk of those carrying the GG genotype (95\% confidence interval (Cl) of odds ratio (OR): 1.01-1.96), and the NIHL risk of the subjects with the CC genotype was 0.93 times the risk of those carrying the $\mathrm{GG}$ genotype $(95 \% \mathrm{Cl}$ of OR: $0.71-1.21)$. After the noise exposure period and noise exposure intensities were stratified, in the co-dominant model, the adjusted OR values for noise intensities of $\leq 85$ was 1.23 (95\%Cl: $0.99-1.53$ ). In the dominant model, the adjusted OR values for noise exposure periods of $\leq 16$ years and noise intensities of $\leq 85$ were 1.88 (95\%Cl: 1.03-3.42) and 1.64 (95\%Cl: 1.12-2.38), respectively.

\footnotetext{
*Correspondence: zhubaoli1965@126.com

${ }^{\dagger}$ Hao Chen, Xuexue Ding and Enming Ding have contributed equally to this research

${ }^{1}$ Center for Global Health, School of Public Health, Nanjing Medical University, Nanjing 210000, Jiangsu, China

Full list of author information is available at the end of the article
}

(C) The Author(s) 2021. Open Access This article is licensed under a Creative Commons Attribution 4.0 International License, which permits use, sharing, adaptation, distribution and reproduction in any medium or format, as long as you give appropriate credit to the original author(s) and the source, provide a link to the Creative Commons licence, and indicate if changes were made. The images or other third party material in this article are included in the article's Creative Commons licence, unless indicated otherwise in a credit line to the material. If material is not included in the article's Creative Commons licence and your intended use is not permitted by statutory regulation or exceeds the permitted use, you will need to obtain permission directly from the copyright holder. To view a copy of this licence, visit http://creativecommons.org/licenses/by/4.0/. The Creative Commons Public Domain Dedication waiver (http://creativeco mmons.org/publicdomain/zero/1.0/) applies to the data made available in this article, unless otherwise stated in a credit line to the data. 
Conclusion: The CC/CG genotype of rs2585405 in the PER1 gene was identified as a potential risk factor for NIHL in Chinese noise-exposed workers, and interaction between rs2585405 and high temperature was found to be associated with $\mathrm{NIHL}$ risk.

Keywords: Cochlear clock genes, PER1, Polymorphism, Noise-induced hearing loss, Susceptibility

\section{Background}

Noise-induced hearing loss (NIHL), which ranks the second among the forms of sensorineural hearing loss worldwide [1], is an occupational health hazard worldwide. NIHL is widely acknowledged as a complicated disease arising from the synergistic effect of inherited factors and environmental factors [2]. The World Health Organization and The National Institute of Occupational Safety and Health have listed NIHL as a research focus. The pathogenesis of NIHL is not completely revealed at the moment. Possible hypotheses for the pathogenesis include the overloaded calcium channels, which stimulates cell apoptosis [3-5]; the production of free radicals or reactive oxygen species [6-8]; and glutamate excitotoxicity, activating signaling pathways that lead to cell death [9]. Variations of NIHL vulnerability exhibited in individuals had been indicated by previous human and animal research $[10,11]$.

The production of circadian rhythm is a process involving cells. The transcription and expression of PERs and $C R Y S$ are irritated by core clock genes CLOCK and $B M A L 1$, the products of PERs and CRYs combined with CLOCK and BMAL1 to inhibit its own transcription and expression $[12,13]$. When the products reach a certain level, inhibition stops, and CLOCK and BMAL1 start a new transcription cycle [14, 15]. Previous studies reported that auditory function could be affected by circadian rhythm [16] and that self-sustaining circadian rhythm exists in the cochlea $[17,18]$. Animal research indicated that the expression of core clock genes could be affected by noise, resulting in the disturbance of circadian rhythm and impairing auditory function [16-18]. Furthermore, some studies revealed that both PER1 and $P E R 2$ were expressed in the cochlea and inferior colliculus $[17,18]$. BDNF and NTF3 belong to the nerve growth factor (NGF) protein family [19], with similar structures [20]. Previous research showed that BDNF and NTF-3 played important roles in the growth and development of spiral ganglion neurons (SGN). During the development of the cochlea, neurotrophic factors regulate the differentiation and survival of neurons [21-24].

Clock genes also play an important role in other human diseases. Loss of the clock gene Per1 promoted oral squamous cell carcinoma progression [25]. The loss of function of Per1 or Per2 led to premature ovarian insufficiency [26]. CRY1 is a tumor-specific regulator of DNA repair related to poor prognosis in patients with prostate cancer [27]. Dysfunctional expression of CRY2 is associated with the susceptibility to depression [28]. A previous study revealed that the association of sleep disturbance and disrupted circadian rhythm led to sudden sensorineural hearing loss (SSNHL), with a lower expression of CRY1 and CRY2 [28]. As far as we know, there has been no research on the correlation between clock genes and NIHL susceptibility.

The purpose of this study was to explore the genetic association between SNPs (single nucleotide polymorphisms) of clock and neurotrophic factor genes and the susceptibility of the Chinese Han population to NIHL, providing clues for research on genetic susceptibility to NIHL.

\section{Material and methods}

\section{Subjects}

The source of subjects for this study was a cohort study that started in 2012. Employees from a textile factory and an energy company in eastward China who received physical health examinations once a year carried by the Jiangsu Provincial Center for Disease Prevention and Control were enlisted for our study in 2019. The inclusion criteria were (1) Chinese Han workers (2) with more than 3 years of noise exposure (hereinafter referred to as "noise exposure"); (3) exposure to noise with other harmful factors (e.g., high temperature, vibration, organic solvents, and carbon monoxide) that may affect the onset of NIHL that was below the occupational exposure limit (GBZ 2.1-2007; GBZ 2.2-2007); and (4) with workplace monitoring data and integral health surveillance data. The exclusion criteria were (1) a history of taking ototoxic drugs and family history of hereditary deafness and other head trauma, (2) people with ear diseases such as ear canal deformity, tympanic membrane perforation. The study was supported by the Ethical Committee of the Jiangsu Provincial Center for Disease Prevention and Control and informed consent was obtained from the research subjects.

\section{Research design}

A nested case-control design was performed in this study. The participants in this study were part of a cohort that started in 2012, which included employees exposed to noise in a textile factory and an energy company in 
eastern China by the Jiangsu Provincial Center for Disease Prevention and Control who had annual follow-up examinations. Our research participants were the 2019 cohort of the study begun in 2012.

\section{Questionnaire investigation}

This study used the noise-induced hearing loss questionnaire designed by the research group. The content of the questionnaire included (1) informed consent; (2) basic information (marriage, sex, age, and education); (3) tobacco and alcohol consumption habits; (4) current and past medical history (e.g., hypertension, diabetes, hyperlipidemia, and ear diseases); and (5) occupational noise exposure history (work years, noise exposure years, and protective measures).

\section{Pure-tone audiometry (PTA) testing and environmental noise measurement}

The pure-tone audiometry was examined applying A Madsen Voyager 522 audiometer (Madsen, Taastrup, Denmark). The binaural hearing test was conducted for all participants after staying away from noise for at least $48 \mathrm{~h}$ to eliminate the effect of temporary hearing threshold shift on the results. Then, each subject was examined at 6 frequencies of 500, 1000, 2000, 3000, 4000, and $6000 \mathrm{~Hz}$, respectively in an soundproof room. The formular for calculating binaural high frequency average hearing threshold is as follows. $i$ is equal to the number of different posts; $T$ refers to consumption of the time at various locations; $L_{A e q, 8} h$ is equal to sequential sound level of $8 \mathrm{~h}$ for different types of work.

\section{Definition of NIHL and control subjects and propensity score matching}

Hearing abnormity and hearing normality were determined on the basis of the Chinese diagnostic criteria applying to occupational noise-induced deafness (GBZ 49-2007). In this study, hearing loss was identified using binaural high-frequency hearing thresholds outside of $25 \mathrm{~dB}(\mathrm{~A})$. Correspondingly, hearing normality was identified by a binaural high-frequency hearing threshold below of $25 \mathrm{~dB}(\mathrm{~A})$. The hearing threshold values were obtained by PTA testing. All participants were divided into NIHL and normal hearing groups. The NIHL individuals refer to noise-exposed individuals with hearing abnormity and the control individuals were determined as noise-exposed individuals with hearing normality. The NIHL patients were selected first, and the controls were matched to the patients according to sex, age, and noise exposure using propensity score matching at a ratio of 1:2. Eventually, 432 NIHL patients and 837 controls were selected from all participants.

\section{Extraction of DNA and selection of SNPs and genotyping}

Peripheral blood $(2 \mathrm{ml})$ of the subjects was drawn into ethylene diamine tetraacetic acid (EDTA) tubes, centri-

Binaural high frequency average hearing threshold

$$
=\frac{\text { left }\left(H L_{3 K H Z}+H L_{4 K H Z}+H L_{6 K H Z}\right)+\operatorname{righ}\left(H L_{3 K H Z}+H L_{4 K H Z}+H L_{6 K H Z}\right)}{6} \text {, }
$$

left is left ear; righ is right ear; $H L$ is missing value of 6 frequencies of 500, 1000, 2000, 3000, 4000, and $6000 \mathrm{~Hz}$, dB.

Noise exposure level was evaluated in each working point using noise dosimeters (Noise-Pro; Quest, Oconomowoc, WI, USA) by the direction of the Chinese National Criteria for Noise at the Workplace. Cumulative noise exposure (CNE) was applied to determine noise exposure level of individuals in this study. The calculation of CNE is based on the 8-h equivalent continuous sound level (A) in the operating points, and the formular for calculating $\mathrm{CNE}$ is as follows.

$$
\mathrm{CNE}=10 \log \left[\frac{1}{\mathrm{~T}_{\text {ref }}} \sum_{\mathrm{i}=1}^{\mathrm{n}}\left(\mathrm{T}_{\mathrm{i}} \times 10^{\mathrm{L}_{\text {Aeq }, 8 \mathrm{~h} / 10}}\right)\right],
$$

$T_{\text {ref }}$ refers to $1 ; n$ is equivalent to overall number of different work position exposed to noise for the workers; fuging $3000 \mathrm{r} / \mathrm{min}$ for $5 \mathrm{~min}$ to extract DNA for genotyping. The isolation of DNA from the peripheral blood was conducted by the QIA cube HT and the QIA amp 96 DNA QIA cube HT Kit (Qiagen, Dusseldorf, Germany). Subsequent to the isolation, the DNA samples were stored at $-20{ }^{\circ} \mathrm{C}$ until use.

The selection of the SNPs in the target genes was from the National Center for Biotechnology Information (NCBI) database (http://www.ncbi.nlm.nih.gov/) and previous literature using the standard of a minimum allele frequency (MAF) of $>0.05$ and present in a Chinese Han population. In accordance with the standard, 12 SNPs met the criteria (rs2253820, rs2585405, rs56386336, rs934945, rs1056560, rs3809236, rs2292910, rs6798, rs11030099, rs7124442, rs6265rs, and 1805149). All these SNPs are located in the functional areas of the chromosomes. 
ABI TaqMan SNP genotyping assays (Applied Biosystems, Foster City, CA, USA) and genotyping probes and primers that designed commercially were adopted to confirm the genotypes of the selected SNPs. The designed commercial products were mixed with TaqMan Universal PCR Master Mix (Roche, Branchburg, NJ, USA) respectively following the attached guideline. After thawing, the extracted DNA were added into mixture. A QuantStudio ${ }^{\mathrm{TM}} 7$ Flex System (Applied Biosystems) was used to perform the genotyping. The results were analyzed using QuantStudio ${ }^{\text {TM }} 7$ Flex System Sequence Detection software version 1.2.3 (Applied Biosystems).

\section{Statistical analyses}

Statistical analysis was conducted with the assistance of SAS 9.4 software. The continuous variables and categorical variables were described as mean $\pm S D$ and percentages respectively. The differences in age, years of exposure to noise, and the intensity of noise exposure between the NIHL and control groups were evaluated using Student's t-tests or paired t-tests. The differences in sex, tobacco and alcohol consumption, and genotype distribution among the two groups were compared using the $x^{2}$ test. Hardy-Weinberg equilibrium in the target SNPs of the participants were calculated by goodnessof-fit $X^{2}$ tests. The odds ratios (ORs) with $95 \%$ confidence intervals $(95 \% \mathrm{CI})$ of the subjects who were different genotypes were defined using conditional logistic regression models adjusted for age, sex, smoking, and drinking. The interaction analysis between rs2585405 and high temperature was performed using MDR 3.0.2 software. Propensity score matching was conducted using R 4.0.5 software. Sensitivity analysis was performed using SAS 9.4 software. The Bonferroni method was used to correct all $P$-values, and statistical significance was determined as $P<0.05$.

\section{Results}

Basic information of study subjects and Hardy-Weinberg tests of selected SNPs

General information (sex and age), life habit characteristics (consumption of tobacco and alcohol, noise exposure, and noise-intensity), and high-frequency hearing threshold shifts are shown in Table 1. No significant difference was found in the general information and lifestyle features between the two groups. However, there was a significant difference in highfrequency hearing threshold shifts between the NIHL and control groups $(P<0.001)$. General information on the selected SNPs and the results of the HardyWeinberg test are shown in Table 2. All selected SNPs had minor allele frequencies of $\geq 5 \%$ and were
Table 1 Demographic characteristics of study subjects

\begin{tabular}{|c|c|c|c|c|c|}
\hline \multirow[t]{2}{*}{ Variables } & \multicolumn{2}{|c|}{ Cases $(n=432)$} & \multicolumn{2}{|c|}{ Controls $(n=837)$} & \multirow[t]{2}{*}{$P$} \\
\hline & $\mathrm{n}$ & $\%$ & $\mathbf{n}$ & $\%$ & \\
\hline \multicolumn{5}{|l|}{ Age (years) } & $0.09^{\mathrm{a}}$ \\
\hline Mean \pm SD & \multicolumn{2}{|l|}{$47.85 \pm 5.48$} & \multicolumn{2}{|l|}{$47.43 \pm 5.38$} & $0.18^{b}$ \\
\hline$\leq 35$ & 13 & 1.0 & 26 & 2.1 & \\
\hline $35-45$ & 89 & 7.0 & 219 & 17.3 & \\
\hline$>45$ & 330 & 26.0 & 592 & 46.7 & \\
\hline \multicolumn{5}{|l|}{ Sex } & $0.40^{\mathrm{a}}$ \\
\hline Male & 392 & 30.9 & 771 & 60.8 & \\
\hline Female & 40 & 3.2 & 66 & 5.2 & \\
\hline \multicolumn{5}{|l|}{ Tobacco use } & $0.93^{\mathrm{a}}$ \\
\hline Now & 194 & 15.3 & 372 & 29.3 & \\
\hline Ever & 170 & 13.4 & 338 & 26.6 & \\
\hline Never & 68 & 5.4 & 127 & 10.0 & \\
\hline \multicolumn{5}{|c|}{ Alcohol consumption } & $0.79^{\mathrm{a}}$ \\
\hline Now & 192 & 15.1 & 356 & 28.1 & \\
\hline Ever & 33 & 2.6 & 63 & 5.0 & \\
\hline Never & 207 & 16.3 & 418 & 32.9 & \\
\hline \multicolumn{5}{|c|}{ Duration of noise exposed work (years) } & $0.75^{\mathrm{a}}$ \\
\hline Mean \pm SD & \multicolumn{2}{|l|}{$23.72 \pm 10.58$} & \multicolumn{2}{|l|}{$23.68 \pm 10.55$} & $0.95^{b}$ \\
\hline$\leq 16$ & 84 & 6.6 & 169 & 13.3 & \\
\hline$>16$ & 348 & 27.4 & 668 & 52.6 & \\
\hline \multicolumn{5}{|c|}{ Noise exposure levels (dB) } & $0.17^{\mathrm{a}}$ \\
\hline$\leq 85$ & 265 & 20.9 & 474 & 37.4 & \\
\hline $85-92$ & 126 & 9.9 & 288 & 22.7 & \\
\hline$>92$ & 41 & 3.2 & 75 & 5.9 & \\
\hline \multicolumn{5}{|c|}{ High frequency hearing thresholds (dB) } & $<0.001^{\mathrm{a}}$ \\
\hline Mean $\pm S D$ & $0.64 \pm 0.48$ & & & 0 & $<0.001^{b}$ \\
\hline$\leq 26$ & 155 & 12.2 & 837 & 66.0 & \\
\hline$>26$ & 277 & 21.4 & 0 & 0.0 & \\
\hline
\end{tabular}

${ }^{\text {a }}$ Students' t-test

${ }^{\mathrm{b}}$ Two-sided $\mathrm{x}^{2}$ test

in Hardy-Weinberg equilibrium (HWE) (P > 0.05), indicating that the sample selected in this study was representative of the group and that the gene frequency of the research objects could represent the gene distribution of the population.

Multivariate analysis of selected SNPs with the risk of NIHL Twelve SNPs were determined in 1269 workers exposed to noise (432 NIHL patients and 837 controls). The results of the genotypes and allele distributions of the twelve SNPs are shown in Table 3. Analysis of the selected SNPs in 4 gene models (codominant, dominant, recessive, and allelic models) showed statistically significant differences in the genotype frequencies of gene PER1 rs2585405 between the cases and controls $(P=0.032$ and $P=0.01$, 
Table 2 General information of selected SNPs and Hardy-Weinberg test

\begin{tabular}{|c|c|c|c|c|c|c|}
\hline Gene & SNP & Alleles & Chromosome & Functional Consequence & MAF database ${ }^{a}$ & $P$ for HWE ${ }^{b}$ \\
\hline \multirow[t]{2}{*}{ PER1 } & rs2253820 & $A / G$ & $17: 8144851$ & splice region variant & 0.44 & 0.954 \\
\hline & rs2585405 & $C / G$ & $17: 8143454$ & missense variant & 0.23 & 0.441 \\
\hline \multirow[t]{2}{*}{ PER2 } & rs56386336 & $A / G$ & $2: 238245307$ & 3'prime UTR variant & 0.09 & 1.000 \\
\hline & rs934945 & $A / G$ & $2: 238246412$ & missense variant & 0.17 & 0.026 \\
\hline \multirow[t]{2}{*}{ CRY1 } & rs1056560 & $\mathrm{G} / \mathrm{T}$ & 12:106991832 & 3'prime UTR variant & 0.47 & 0.402 \\
\hline & rs3809236 & $C / T$ & 12:107093269 & 5'prime UTR variant & 0.20 & 0.892 \\
\hline \multirow[t]{2}{*}{$C R Y 2$} & rs2292910 & $\mathrm{C} / \mathrm{A}$ & $11: 45882062$ & 3'prime UTR variant & 0.42 & 0.994 \\
\hline & rs6798 & $C / T$ & $11: 45882926$ & 3'prime UTR variant & 0.24 & 0.892 \\
\hline \multirow[t]{3}{*}{$B D N F$} & rs11030099 & $\mathrm{A} / \mathrm{C}$ & $11: 27656036$ & 3'prime UTR variant & 0.23 & 0.205 \\
\hline & rs7124442 & $\mathrm{C} / \mathrm{T}$ & $11: 27655494$ & 3'prime UTR variant & 0.33 & 0.654 \\
\hline & rs6265 & $A / G$ & $11: 27658369$ & missense variant & 0.20 & 0.264 \\
\hline NTF3 & rs1805149 & $A / G$ & 12:5494441 & missense variant & 0.12 & 0.592 \\
\hline
\end{tabular}

a Data from NCBI dbSNP

${ }^{b} P$ value of Hardy-Weinberg test

respectively) in the codominant and dominant models. In the codominant model, the GC and CC genotypes were risk factors for the onset of NIHL, and individuals with GC or CC had an increased risk of NIHL $(\mathrm{OR}=1.41$, 95\%CI: $1.01-1.96$, OR $=0.93$, 95\%CI: 0.71-1.21, respectively) by logistic regression analysis after adjusting for age, sex, and alcohol and tobacco consumption habits. In the dominant model, logistic regression analysis after adjusting for age, sex, and alcohol and tobacco consumption habits demonstrated that individuals with the $\mathrm{GC} /$ $\mathrm{CC}$ genotype had a significantly increased risk for NIHL $(\mathrm{OR}=1.47,95 \% \mathrm{CI}$ : 1.10-1.97). Thus, our data revealed that the gene PER1 rs2585405 may have a significant association with increased NIHL susceptibility.

\section{Stratified analysis of rs 2585405 polymorphism and NIHL risk}

The results of stratified analysis of rs 2585405 polymorphism and NIHL risk are shown in Table 4. In the group with noise exposure levels of $\leq 85 \mathrm{~dB}$, the distribution of rs2585405 genotypes was significantly different between the cases and controls $(P=0.03)$, and individuals with GC + CC genotypes had an increased NIHL risk $(\mathrm{OR}=1.23,95 \% \mathrm{CI}$ : $0.99-1.53)$. In the dominant model, in the group with noise exposures of $\leq 16$ and noise intensities of $\leq 85 \mathrm{~dB}$, statistically significant differences were found in the rs 258540 genotype distributions between the cases and controls in $(P=0.04$ and $P=0.01$, respectively), and the NIHL risk of individuals with GC/CC genotypes between the cases and controls increased from 1.47 times before stratification $(\mathrm{OR}=1.47,95 \% \mathrm{CI}$ : 1.10 $1.97)$ to 1.88 times $(\mathrm{OR}=1.88,95 \% \mathrm{CI}: 1.03-3.42)$ and 1.64 times $(\mathrm{OR}=1.64,95 \% \mathrm{CI}: 1.12-2.38)$.

\section{Multifactor dimensionality reduction analysis of the interaction between rs2585405 and high temperature}

The results of the multifactor dimensionality (MDR) analysis of the interaction between rs2585405 and high temperature are shown in Table 5 and Fig. 1. The results indicated that the rs $2585405^{*}$ high temperature and rs6265"rs934945"rs1805149 models were associated with NIHL risk ( $\mathrm{OR}=1.61$ and 1.80 , respectively, $P<0.001$ ), which indicated that high temperature and rs2585405 were interactive risk factors for NIHL.

\section{Sensitivity analysis}

The results of the sensitivity analysis were consistent with our analysis. The sensitivity analysis results are attached to Additional files 1-4.

\section{Discussion}

SNPs are considered to be universal genetic variations in the human genome, and there are as many as 15 million SNPs among all humans [29]. SNPs are unevenly distributed in the genome. The frequency of SNPs in non-coding regions is higher than that in the gene coding regions. The present methods of detecting SNPs mainly include denaturing gradient gel electrophoresis, single-strand conformational polymorphism analysis, cleaved amplified polymorphic sequence assays, denaturing gradient gel electrophoresis, and allele-specific PCR (Taq-Man SNP genotype-PCR).

In this study, a large sample of Han nationality noise workers was preliminarily analyzed for the association between a total of twelve SNPs among clock and nerve growth factor genes and susceptibility to NIHL. 
Table 3 Distribution of selected SNPs and the association with NIHL

\begin{tabular}{|c|c|c|c|c|c|c|c|c|c|}
\hline \multirow[t]{2}{*}{ Gene } & \multirow[t]{2}{*}{ Genetic models } & \multirow[t]{2}{*}{ Genotypes } & \multicolumn{2}{|l|}{ Cases } & \multicolumn{2}{|l|}{ Controls } & \multirow[t]{2}{*}{$P^{a}$} & \multirow[t]{2}{*}{ FDR } & \multirow{2}{*}{$\begin{array}{l}\text { Adjusted OR } \\
(95 \% \mathrm{Cl})^{\mathrm{b}}\end{array}$} \\
\hline & & & $n=432$ & $\%$ & $n=837$ & $\%$ & & & \\
\hline \multirow[t]{20}{*}{ PER1 } & rs2585405 & & & & & & & & \\
\hline & Codominant & GG & 98 & 22.7 & 140 & 16.7 & 0.03 & 0.36 & 1.00 (Ref.) \\
\hline & & GC & 198 & 45.8 & 425 & 50.8 & & & $1.41(1.01-1.96)$ \\
\hline & & $\mathrm{CC}$ & 136 & 31.5 & 272 & 32.5 & & & $0.93(0.71-1.21)$ \\
\hline & Dominant & GG & 98 & 22.7 & 140 & 16.7 & 0.01 & 0.12 & 1.00 (Ref.) \\
\hline & & $\mathrm{GC} / \mathrm{CC}$ & 334 & 77.3 & 697 & 83.3 & & & $1.47(1.10-1.97)$ \\
\hline & Recessive & $\mathrm{GG} / \mathrm{GC}$ & 296 & 68.5 & 565 & 67.5 & 0.71 & 0.97 & 1.00 (Ref.) \\
\hline & & CC & 136 & 31.5 & 272 & 32.5 & & & $1.05(0.82-1.34)$ \\
\hline & Alleles & G & 194 & 31.0 & 705 & 55.6 & 0.09 & 0.88 & 1.00 (Ref.) \\
\hline & & C & 470 & 37.0 & 969 & 76.4 & & & $1.15(0.98-1.36)$ \\
\hline & rs 2253820 & & & & & & & & \\
\hline & Codominant & GG & 44 & 10.2 & 96 & 11.5 & 0.45 & 0.98 & 1.00 (Ref.) \\
\hline & & $A G$ & 196 & 45.4 & 399 & 47.7 & & & $0.82(0.55-1.22)$ \\
\hline & & AA & 192 & 44.4 & 342 & 40.9 & & & $0.87(0.68-1.12)$ \\
\hline & Dominant & GG & 44 & 10.2 & 96 & 11.5 & 0.49 & 0.95 & 1.00 (Ref.) \\
\hline & & $\mathrm{AA} / \mathrm{AG}$ & 388 & 89.8 & 741 & 88.5 & & & $0.88(0.60-1.28)$ \\
\hline & Recessive & $A G / G G$ & 240 & 55.6 & 495 & 59.1 & 0.22 & 0.97 & 1.00 (Ref.) \\
\hline & & AA & 192 & 44.4 & 342 & 40.9 & & & $0.86(0.68-1.09)$ \\
\hline & Alleles & G & 284 & 22.4 & 591 & 46.6 & 0.22 & 0.88 & 1.00 (Ref.) \\
\hline & & A & 580 & 45.7 & 1083 & 85.3 & & & $0.89(0.75-1.07)$ \\
\hline \multirow[t]{20}{*}{ PER2 } & rs56386336 & & & & & & & & \\
\hline & Codominant & GG & 332 & 76.9 & 661 & 79.0 & 0.50 & 0.98 & 1.00 (Ref.) \\
\hline & & $A G$ & 96 & 22.2 & 165 & 19.7 & & & $1.26(0.40-4.02)$ \\
\hline & & $\mathrm{AA}$ & 4 & 0.9 & 11 & 1.3 & & & $1.47(0.45-4.78)$ \\
\hline & Dominant & GG & 332 & 76.9 & 661 & 79.0 & 0.39 & 0.95 & 1.00 (Ref.) \\
\hline & & $A G / G G$ & 100 & 23.1 & 176 & 21.0 & & & $0.88(0.66-1.16)$ \\
\hline & Recessive & $\mathrm{GG} / \mathrm{AG}$ & 428 & 99.1 & 826 & 98.7 & 0.54 & 0.97 & 1.00 (Ref.) \\
\hline & & $\mathrm{AA}$ & 4 & 0.9 & 11 & 1.3 & & & $1.31(0.41-4.15)$ \\
\hline & Alleles & G & 760 & 59.9 & 1487 & 117.1 & 0.52 & 0.90 & 1.00 (Ref.) \\
\hline & & A & 104 & 8.2 & 187 & 14.7 & & & $1.10(0.85-1.43)$ \\
\hline & rs934945 & & & & & & & & \\
\hline & Codominant & GG & 242 & 56.0 & 458 & 54.7 & 0.75 & 0.99 & 1.00 (Ref.) \\
\hline & & $A G$ & 157 & 36.3 & 305 & 36.4 & & & $1.19(0.77-1.85)$ \\
\hline & & $\mathrm{AA}$ & 33 & 7.6 & 74 & 8.8 & & & $1.16(0.73-1.82)$ \\
\hline & Dominant & GG & 242 & 56.0 & 458 & 54.7 & 0.66 & 0.95 & 1.00 (Ref.) \\
\hline & & $\mathrm{AG} / \mathrm{AA}$ & 190 & 44.0 & 379 & 45.3 & & & $1.06(0.84-1.34)$ \\
\hline & Recessive & $A G / G G$ & 399 & 92.4 & 763 & 91.2 & 0.47 & 0.97 & 1.00 (Ref.) \\
\hline & & AA & 33 & 7.6 & 74 & 8.8 & & & $1.18(0.77-1.81)$ \\
\hline & Alleles & G & 641 & 50.5 & 1221 & 96.2 & 0.50 & 0.90 & 1.00 (Ref.) \\
\hline & & A & 223 & 17.6 & 453 & 35.7 & & & $0.94(0.78-1.13)$ \\
\hline \multirow[t]{8}{*}{ CRY1 } & rs1056560 & & & & & & & & \\
\hline & Codominant & GG & 25 & 5.8 & 54 & 6.5 & 0.37 & 0.98 & 1.00 (Ref.) \\
\hline & & GT & 143 & 33.1 & 306 & 36.6 & & & $0.83(0.51-1.37)$ \\
\hline & & $\mathrm{TT}$ & 264 & 61.1 & 477 & 57.0 & & & $0.84(0.66-1.08)$ \\
\hline & Dominant & GG & 25 & 5.8 & 54 & 6.5 & 0.64 & 0.95 & 1.00 (Ref.) \\
\hline & & $\mathrm{GT} / \mathrm{TT}$ & 407 & 94.2 & 783 & 93.5 & & & $0.89(0.54-1.45)$ \\
\hline & Recessive & GT/GG & 168 & 38.9 & 360 & 43.0 & 0.16 & 0.97 & 1.00 (Ref.) \\
\hline & & $\mathrm{TT}$ & 264 & 61.1 & 477 & 57.0 & & & $0.84(0.66-1.07)$ \\
\hline
\end{tabular}


Table 3 (continued)

\begin{tabular}{|c|c|c|c|c|c|c|c|c|c|}
\hline \multirow[t]{2}{*}{ Gene } & \multirow[t]{2}{*}{ Genetic models } & \multirow[t]{2}{*}{ Genotypes } & \multicolumn{2}{|l|}{ Cases } & \multicolumn{2}{|l|}{ Controls } & \multirow[t]{2}{*}{$P^{\mathrm{a}}$} & \multirow[t]{2}{*}{ FDR } & \multirow{2}{*}{$\begin{array}{l}\text { Adjusted OR } \\
(95 \% \mathrm{Cl})^{\mathrm{b}}\end{array}$} \\
\hline & & & $n=432$ & $\%$ & $\mathrm{n}=837$ & $\%$ & & & \\
\hline & Alleles & G & 193 & 15.2 & 414 & 326 & 0.18 & 0.88 & 1.00 (Ref.) \\
\hline & & $\mathrm{T}$ & 671 & 52.9 & 1260 & 99.3 & & & $0.88(0.72-1.06)$ \\
\hline & rs3809236 & & & & & & & & \\
\hline & Codominant & $\mathrm{CC}$ & 276 & 63.9 & 514 & 61.4 & 0.59 & 0.98 & 1.00 (Ref.) \\
\hline & & $\mathrm{TC}$ & 139 & 32.2 & 282 & 33.7 & & & $1.32(0.73-2.37)$ \\
\hline & & $\mathrm{TT}$ & 17 & 3.9 & 41 & 4.9 & & & $1.21(0.66-2.22)$ \\
\hline & Dominant & $\mathrm{CC}$ & 276 & 63.9 & 514 & 61.4 & 0.39 & 0.95 & 1.00 (Ref.) \\
\hline & & $\mathrm{TC} / \mathrm{TT}$ & 156 & 36.1 & 323 & 38.6 & & & $1.11(0.87-1.42)$ \\
\hline & Recessive & $\mathrm{TC} / \mathrm{CC}$ & 415 & 96.1 & 796 & 95.1 & 0.44 & 0.97 & 1.00 (Ref.) \\
\hline & & $\mathrm{TT}$ & 17 & 3.9 & 41 & 4.9 & & & $1.28(0.72-2.29)$ \\
\hline & Alleles & C & 691 & 54.5 & 1310 & 103.2 & 0.31 & 0.90 & 1.00 (Ref.) \\
\hline & & $\mathrm{T}$ & 173 & 13.6 & 364 & 28.7 & & & $0.90(0.73-1.10)$ \\
\hline \multirow[t]{20}{*}{ CRY2 } & rs2292910 & & & & & & & & \\
\hline & Codominant & CC & 36 & 8.3 & 75 & 9.0 & 0.90 & 0.99 & 1.00 (Ref.) \\
\hline & & $A C$ & 180 & 41.7 & 340 & 40.6 & & & $0.93(0.61-1.43)$ \\
\hline & & $\mathrm{AA}$ & 216 & 50.0 & 422 & 50.4 & & & $1.04(0.81-1.32)$ \\
\hline & Dominant & $\mathrm{CC}$ & 36 & 8.3 & 75 & 9.0 & 0.71 & 0.95 & 1.00 (Ref.) \\
\hline & & AC/AA & 396 & 91.7 & 762 & 91.0 & & & $0.92(0.61-1.39)$ \\
\hline & Recessive & $\mathrm{AC} / \mathrm{CC}$ & 216 & 50.0 & 415 & 49.6 & 0.89 & 0.97 & 1.00 (Ref.) \\
\hline & & AA & 216 & 50.0 & 422 & 50.4 & & & $1.02(0.81-1.28)$ \\
\hline & Alleles & C & 252 & 19.9 & 490 & 38.6 & 0.96 & 0,99 & 1.00 (Ref.) \\
\hline & & A & 612 & 48.2 & 1184 & 93.3 & & & $0.99(0.83-1.19)$ \\
\hline & rs6798 & & & & & & & & \\
\hline & Codominant & $\mathrm{CC}$ & 82 & 19.0 & 173 & 20.7 & 0.60 & 0.98 & 1.00 (Ref.) \\
\hline & & $\mathrm{TC}$ & 218 & 50.5 & 398 & 47.6 & & & $0.96(0.69-1.35)$ \\
\hline & & $\mathrm{TT}$ & 132 & 30.6 & 266 & 31.8 & & & $1.10(0.84-1.44)$ \\
\hline & Dominant & CC & 82 & 19.0 & 173 & 20.7 & 0.48 & 0.95 & 1.00 (Ref.) \\
\hline & & $\mathrm{TC} / \mathrm{TT}$ & 350 & 81.0 & 664 & 79.3 & & & $0.91(0.68-1.22)$ \\
\hline & Recessive & $\mathrm{TC} / \mathrm{CC}$ & 300 & 69.4 & 571 & 68.2 & 0.66 & 0.97 & 1.00 (Ref.) \\
\hline & & $\mathrm{TT}$ & 132 & 30.6 & 266 & 31.8 & & & $1.06(0.82-1.37)$ \\
\hline & Alleles & $C$ & 382 & 30.1 & 744 & 58.6 & 0.91 & 0.99 & 1.00 (Ref.) \\
\hline & & $\mathrm{T}$ & 482 & 38.0 & 930 & 73.3 & & & $0.99(0.84-1.17)$ \\
\hline \multirow[t]{16}{*}{$B D N F$} & rs11030099 & & & & & & & & \\
\hline & Codominant & $\mathrm{CC}$ & 139 & 32.2 & 250 & 29.9 & 0.65 & 0.98 & 1.00 (Ref.) \\
\hline & & $A C$ & 195 & 45.1 & 398 & 47.6 & & & $1.08(0.78-1.48)$ \\
\hline & & AA & 98 & 22.7 & 189 & 22.6 & & & $0.95(0.70-1.28)$ \\
\hline & Dominant & CC & 139 & 32.2 & 250 & 29.9 & 0.40 & 0.95 & 1.00 (Ref.) \\
\hline & & $\mathrm{AC} / \mathrm{AA}$ & 293 & 67.8 & 587 & 70.1 & & & $1.11(0.87-1.43)$ \\
\hline & Recessive & $\mathrm{AC} / \mathrm{CC}$ & 334 & 77.3 & 648 & 77.4 & 0.97 & 0.97 & 1.00 (Ref.) \\
\hline & & $\mathrm{AA}$ & 98 & 22.7 & 189 & 22.6 & & & $1.00(0.76-1.32)$ \\
\hline & Alleles & $C$ & 473 & 37.3 & 898 & 70.8 & 0.60 & 0.90 & 1.00 (Ref.) \\
\hline & & A & 391 & 30.8 & 776 & 61.2 & & & $0.96(0.82-1.12)$ \\
\hline & rs7124442 & & & & & & & & \\
\hline & Codominant & $\mathrm{CC}$ & 1 & 0.2 & 2 & 0.2 & 0.95 & 0.99 & 1.00 (Ref.) \\
\hline & & $\mathrm{TC}$ & 58 & 13.4 & 107 & 12.8 & & & $0.89(0.08-9.93)$ \\
\hline & & $\mathrm{TT}$ & 373 & 86.3 & 728 & 87.0 & & & $1.06(0.75-1.50)$ \\
\hline & Dominant & $\mathrm{CC}$ & 1 & 0.2 & 2 & 0.2 & 1.00 & 1.00 & 1.00 (Ref.) \\
\hline & & $\mathrm{TC} / \mathrm{TT}$ & 431 & 99.8 & 835 & 99.8 & & & $0.88(0.08-9.85)$ \\
\hline
\end{tabular}


Table 3 (continued)

\begin{tabular}{|c|c|c|c|c|c|c|c|c|c|}
\hline \multirow[t]{2}{*}{ Gene } & \multirow[t]{2}{*}{ Genetic models } & \multirow[t]{2}{*}{ Genotypes } & \multicolumn{2}{|l|}{ Cases } & \multicolumn{2}{|l|}{ Controls } & \multirow[t]{2}{*}{$P^{\mathrm{a}}$} & \multirow[t]{2}{*}{ FDR } & \multirow{2}{*}{$\begin{array}{l}\text { Adjusted OR } \\
(95 \% \mathrm{Cl})^{\mathrm{b}}\end{array}$} \\
\hline & & & $n=432$ & $\%$ & $n=837$ & $\%$ & & & \\
\hline & Recessive & $\mathrm{TC} / \mathrm{CC}$ & 59 & 13.7 & 109 & 13.0 & 0.75 & 0.97 & 1.00 (Ref.) \\
\hline & & $\mathrm{TT}$ & 373 & 86.3 & 728 & 87.0 & & & $1.06(0.75-1.49)$ \\
\hline & Alleles & C & 60 & 4.7 & 111 & 8.7 & 0.77 & 0.99 & 1.00 (Ref.) \\
\hline & & $\mathrm{T}$ & 804 & 63.4 & 1563 & 123.2 & & & $1.05(0.75-1.46)$ \\
\hline & rs6265 & & & & & & & & \\
\hline & Codominant & GG & 125 & 28.9 & 240 & 28.7 & 0.99 & 0.99 & 1.00 (Ref.) \\
\hline & & $A G$ & 204 & 47.2 & 399 & 47.7 & & & $1.00(0.73-1.38)$ \\
\hline & & AA & 103 & 23.8 & 198 & 23.7 & & & $0.99(0.74-1.33)$ \\
\hline & Dominant & GG & 125 & 28.9 & 240 & 28.7 & 0.92 & 1.00 & 1.00 (Ref.) \\
\hline & & $\mathrm{AA} / \mathrm{AG}$ & 307 & 71.1 & 597 & 71.3 & & & $1.01(0.78-1.30)$ \\
\hline & Recessive & $\mathrm{AG} / \mathrm{GG}$ & 329 & 76.2 & 639 & 76.3 & 0.94 & 0.97 & 1.00 (Ref.) \\
\hline & & AA & 103 & 23.8 & 198 & 23.7 & & & $0.99(0.76-1.31)$ \\
\hline & Alleles & G & 454 & 35.8 & 879 & 69.3 & 0.99 & 0.99 & 1.00 (Ref.) \\
\hline & & A & 410 & 32.3 & 795 & 62.6 & & & $1.00(0.85-1.17)$ \\
\hline \multirow[t]{10}{*}{ NTF3 } & rs1805149 & & & & & & & & \\
\hline & Codominant & $\mathrm{AA}$ & 97 & 22.5 & 188 & 22.5 & 0.54 & 0.98 & 1.00 (Ref.) \\
\hline & & $A G$ & 67 & 15.5 & 111 & 13.3 & & & $1.02(0.76-1.36)$ \\
\hline & & GG & 268 & 62.0 & 538 & 64.3 & & & $1.20(0.86-1.69)$ \\
\hline & Dominant & $\mathrm{AA}$ & 97 & 22.5 & 188 & 22.5 & 1.00 & 1.00 & 1.00 (Ref.) \\
\hline & & $A G / G G$ & 335 & 77.5 & 649 & 77.5 & & & $0.98(0.74-1.30)$ \\
\hline & Recessive & $\mathrm{AG} / \mathrm{AA}$ & 164 & 38.0 & 299 & 35.7 & 0.43 & 0.97 & 1.00 (Ref.) \\
\hline & & GG & 268 & 62.0 & 538 & 64.3 & & & $0.50(1.09-0.85)$ \\
\hline & Alleles & $A$ & 261 & 20.6 & 487 & 38.4 & 0.56 & 0.90 & 1.00 (Ref.) \\
\hline & & G & 603 & 47.5 & 1187 & 93.6 & & & $1.02(0.89-1.18)$ \\
\hline
\end{tabular}

${ }^{a}$ Two-sided $x^{2}$ test

${ }^{\mathrm{b}}$ Adjusted for age, sex, alcohol, and tobacco use in a logistic regression model

The results showed that the distribution of the PER1 gene rs2585405 genotypes between the case and control groups was statistical different in both the co-dominant model and the dominant model. The risk of NIHL in individuals with the GC/CC genotype was 0.47 times that of people with the GG genotype (adjusted $\mathrm{OR}=0.47$, 95\%CI: 1.10-1.97), indicating that the GC/CC genotype at rs2585405 may be a risk factor for the onset of NIHL.

Sensory information is transmitted from the cochlea to the brainstem by spiral ganglion neurons that are susceptible to noise, senile, and underlying genetic diseases [30]. Evidence has shown that neurotrophic factors played an important role in the treatment of hearing loss. Studies have shown that $B D N F$ and NTF-3 were essential for establishing synapses and maintaining hearing function throughout life [31, 32]. Unfortunately, the SNPs screened in $B D N F$ and NTF-3 in this study did not identify any sites related to NIHL susceptibility.

In the research of Siwei Chen et al., the De novo missense variants in Autism Spectrum Disorder (ASD) patients could impact the risk of ASD by destroying interaction of protein-protein, which is a universal phenomenon in various diseases [33, 34].Dual-oxidase maturation factor 2 (DUOXA2) is a component of thyroid hydrogen peroxidase $(\mathrm{H} 2 \mathrm{O} 2)$ generator, which is essential to hormone synthesis. Congenital hypothyroidism happened with the genetic defect of DUOXA2 causing the damage of $\mathrm{H} 2 \mathrm{O} 2$ production system. A novel DUOXA2 missense mutation (I26M) causes goiter that affected $\mathrm{H} 2 \mathrm{O} 2$ production but did not alter protein expression levels $[35,36]$.

Missense mutation refers to a change in a codon that encodes a certain amino acid to a codon that encodes for another amino acid after a base substitution, which changes the type and sequence of amino acids in the polypeptide chain. As a result of missense mutations, the polypeptide chain usually loses its original function. Many protein abnormalities are caused by missense mutations [37]. Studies had reported that a missense variant in PER2 was related to delayed sleep-wake phase disorder in a Japanese population [38]. A previous study indicated that a missense variant of PER1 rs2585405 was 
Table 4 Stratified analyses of rs2585405 polymorphism and NIHL risk

\begin{tabular}{|c|c|c|c|c|c|c|}
\hline \multirow[t]{2}{*}{ Group } & \multirow[t]{2}{*}{ Genotype } & \multicolumn{2}{|c|}{ noise exposed (years) } & \multicolumn{3}{|c|}{ Expose level with noise (dB) } \\
\hline & & $\leq 16$ & $>16$ & $\leq 85$ & $85-92$ & $>92$ \\
\hline \multirow[t]{3}{*}{ Case } & GG & 27 & 71 & 63 & 26 & 9 \\
\hline & GC & 33 & 165 & 124 & 59 & 15 \\
\hline & $\mathrm{CC}$ & 24 & 112 & 78 & 41 & 17 \\
\hline \multirow[t]{3}{*}{ Control } & GG & 34 & 106 & 76 & 55 & 9 \\
\hline & GC & 80 & 345 & 248 & 139 & 38 \\
\hline & $\mathrm{CC}$ & 55 & 217 & 150 & 94 & 28 \\
\hline$P^{a}$ & & 0.11 & 0.17 & 0.03 & 0.93 & 0.23 \\
\hline Adjusted OR & & 1.33 & 1.11 & 1.23 & 1.02 & 1.07 \\
\hline$(95 \% \mathrm{Cl})^{\mathrm{b}}$ & & $(0.93-1.90)$ & $(0.92-1.34)$ & $(0.99-1.53)$ & $(0.76-1.37)$ & $(0.61-1.87)$ \\
\hline \multirow[t]{2}{*}{ Case } & GG & 27 & 71 & 63 & 26 & 9 \\
\hline & $\mathrm{GC} / \mathrm{CC}$ & 57 & 277 & 202 & 100 & 32 \\
\hline \multirow[t]{2}{*}{ Control } & GG & 34 & 106 & 76 & 55 & 9 \\
\hline & GC/CC & 135 & 562 & 398 & 233 & 66 \\
\hline$p^{a}$ & & 0.04 & 0.07 & 0.01 & 0.72 & 0.16 \\
\hline Adjusted OR & & 1.88 & 1.37 & 1.64 & 1.10 & 1.92 \\
\hline$(95 \% \mathrm{Cl})^{b}$ & & $(1.03-3.42)$ & $(0.98-1.91)$ & $(1.12-2.38)$ & $(0.65-1.85)$ & $(0.67-5.52)$ \\
\hline
\end{tabular}

${ }^{\text {a Two-sided }} x^{2}$ test

${ }^{\mathrm{b}}$ Adjusted for age, sex, alcohol and tobacco use in a logistic regression model

Table 5 Multifactor dimensionality reduction analysis of the interaction between rs2585405 and high temperature

\begin{tabular}{llllll}
\hline Models & $\begin{array}{l}\text { Training balanced } \\
\text { accuracy }\end{array}$ & $\begin{array}{l}\text { Testing balanced } \\
\text { accuracy }\end{array}$ & $\begin{array}{l}\text { Cross svalidation } \\
\text { consistency }\end{array}$ & $\boldsymbol{P}$ & OR (95\%Cl) \\
\hline rs2585405*High temperature & 0.559 & 0.533 & $7 / 10$ & $<0.0001$ & $1.61(1.27-2.05)$ \\
rs6265*rs934945*rs1805149 & 0.579 & 0.501 & $3 / 10$ & $<0.0001$ & $1.80(1.42-2.28)$ \\
\hline
\end{tabular}

associated with the susceptibility to prostate cancer [39]. However, there have been no studies on the correlation between clock genes and NIHL susceptibility. Therefore, in future research, the relevant mechanism of this locus can be explored and more clues on the influence of clock genes on NIHL can be revealed.

The circadian rhythm process depends upon a transcriptional feedback loop initiated by the heterodimeric factor CLOCK: BMAL1 [40]. CLOCK: BMAL1 activates the transcription and expression of core clock genes Period (Per1, Per2, Per3) and Cryptochrome (Cry1 and Cry2). With sufficient expression products, PERs and $C R Y S$ form a complex to inhibit the transcription process mediated by CLOCK: BMAL1 [41-44]. In animal research, the expression of clock genes was inhibited when the circadian rhythm in mice cochlea was disturbed. In addition, mice with normal circadian rhythms in a noisy environment showed higher threshold changes [45]. It had been reported that patients with sudden sensorineural hearing loss showed changes in biological clock genes [46]. These clues suggest that in the prevention strategies of NIHL patients, workers who are exposed to night shift noise should pay more attention to changes in hearing levels.

The research about the interaction between environmental factors and biological factors is essential to reveal response of specific SNPs to environmental factors. As an important biological factor in post-transcriptional processes, mRNA regulates various biological processes [4750]. N4-Acetylcytidine was found being existing broadly on mRNA, which improved stability of mRNA and efficiency of protein translation [51-53]. Some studies have shown that mRNA plays an important role in the occurrence and development of hearing loss [47]. It had been suggested that the networks of microRNA-messenger RNA interactions existed in age-related hearing loss on the basis of researches in aging mice $[54,55]$.

As far as we know, this study is the first to investigate the correlation between clock gene polymorphisms and NIHL susceptibility, but the study had some shortcomings. First, although the number of samples in our study was comparatively large compared to previous studies, 

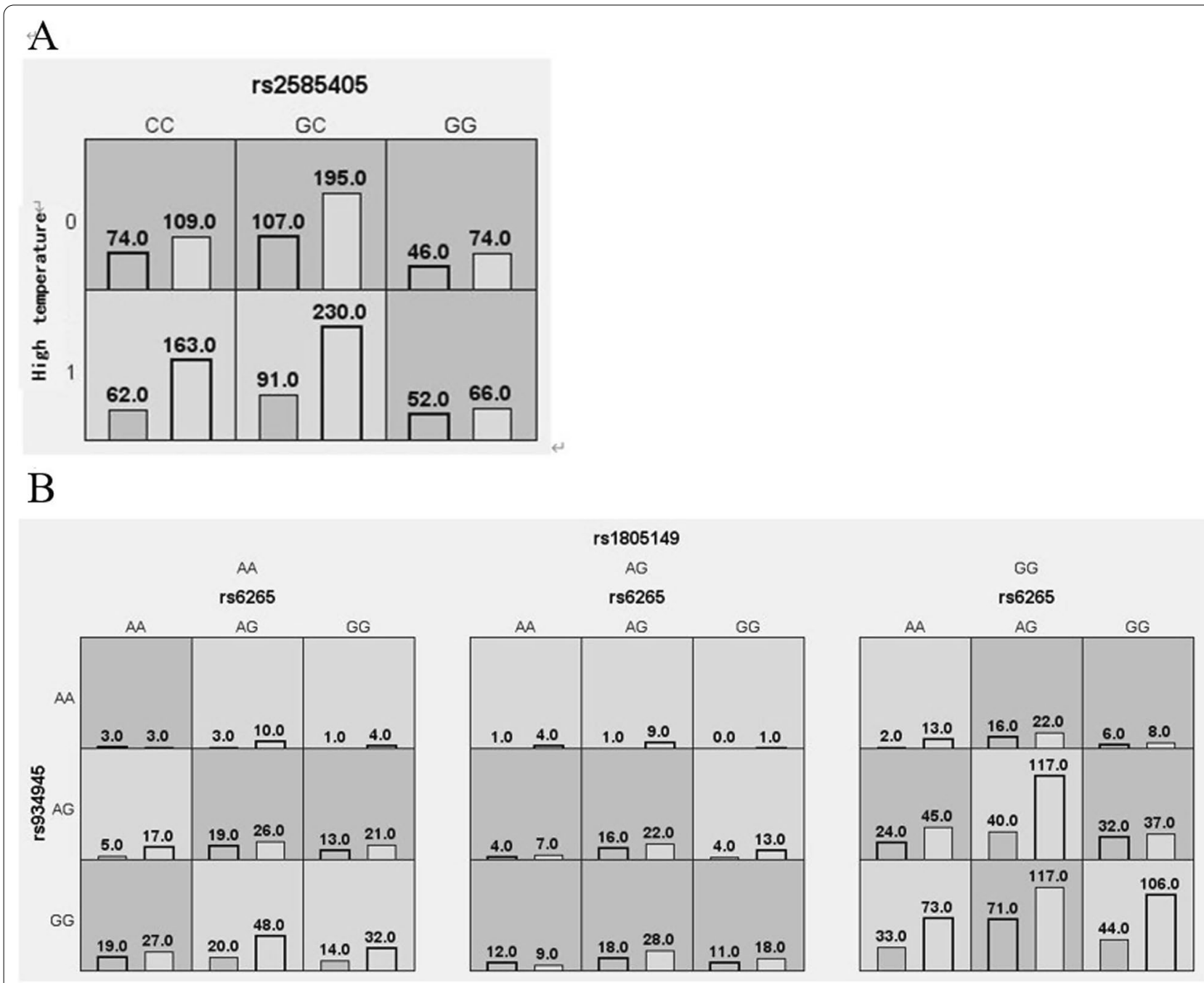

Fig. 1 Graph model of the interaction between rs2585405 and high temperature (A rs2585405*High temperature model, B rs6265*rs934945*rs 1805149 model). Dark gray and light gray boxes presented the high- and low-risk factor combinations, respectively. Left bars within each box represented case while the right bars represented control. The heights of the bars are proportional to the sum of samples in each group

the power of the statistical test was not adequate to confirm the small biological effects of a single SNP possibility. Therefore, larger sample sizes and cohort studies are needed to confirm the impact of clock gene polymorphisms on NIHL risk in the future. Secondly, the subjects in this case-control study were limited to a Chinese population, the trans-ethnic meta-analysis and subgroup meta-analysis according to ethnicity, sex, gene-dosage, and age, based on the selected SNPs was not available to conducted for the limited genotype data [56-60]. Therefore, our results may be more applicable to the Chinese Han population, but the application to other populations may be limited. Thirdly, although the statistical significance between rs2585405 and NIHL susceptibility had been found in our study, it is still not able to determine the disease by the variant. So, the machine-learning model is relatively essential to predict disease risk based the significant SNP in the future research $[61,62]$. Fourthly, the causality of the genetic variant in the development of noise-induced hearing loss was not revealed clearly in our research. So, the genotype data can be integrated with eQTL from GTEX or pQTLs to explore whether the polymorphisms in these genes are causally triggering the development of noise-induced hearing loss through mediating the expression of these genes in specific tissues in further study [63-66]. 


\section{Conclusions}

In conclusion, the $\mathrm{G}$ allele of the PER 1 gene rs 2585405 was associated with NIHL risk, and the site interacts with the factor of high temperature. Therefore, our results indicated that PER1 rs2585405 may play a key role in NIHL and may serve as a biomarker for workers exposed to noise. In addition, this research provides a new research direction that an imbalance in circadian rhythm can impair auditory function.

\section{Supplementary Information}

The online version contains supplementary material available at https://doi. org/10.1186/s12920-021-01075-x.

Additional file 1 Table 6 Sensitivity analysis results of deleting age. The results of sensitivity analysis adjusted for sex, alcohol and tobacco use in a logistic regression model.

Additional file $\mathbf{2}$ Table 7 Sensitivity analysis results of deleting tobacco. The results of sensitivity analysis adjusted for age, sex and alcohol use in a logistic regression model.

Additional file $\mathbf{3}$ Table 8 The results of sensitivity analysis in stratified analyses deleting age. The results of sensitivity analysis in stratified analyses Adjusted for sex, alcohol and tobacco use in a logistic regression model.

Additional file $\mathbf{4}$ Table 9 The results of sensitivity analysis in stratified analyses deleting tobacco. The results of sensitivity analysis in stratified analyses adjusted for age, sex and alcohol use in a logistic regression model.

\section{Acknowledgements}

Thanks to all authors for their contributions to this article.

\section{Authors' contributions}

Conception and design: ED; Administrative support: BZ; Provision of study materials or patients: HW, MC, GY; Collection and assembly of data: HW, MC, GY; Data analysis and interpretation: HC, ED; Manuscript writing: HC. All authors read and approved the final manuscript.

\section{Funding}

This work was supported by Jiangsu Province's Outstanding Medical Academic Leader program (CXTDA2017029).

Availability of data and materials

https://github.com/EnminDing/PER1.

\section{Declarations}

Ethics approval and consent to participate

The study was approved by the Ethical Committee of the Jiangsu Provincial Center for Disease Prevention and Control and informed consent was obtained from the research subjects.

\section{Consent for publication}

Not applicable.

\section{Competing interests}

The authors declare that they have no competing interests.

\section{Author details}

${ }^{1}$ Center for Global Health, School of Public Health, Nanjing Medical University, Nanjing 210000, Jiangsu, China. ${ }^{2}$ Institute of Occupational Disease Prevention, Jiangsu Province Center for Disease Prevention and Control, Nanjing 21009, Jiangsu, China. ${ }^{3}$ Key Laboratory of Environmental Medicine Engineering of Ministry of Education, School of Public Health, Southeast University, Nanjing 210003, Jiangsu, China.

Received: 2 August 2021 Accepted: 31 August 2021

Published online: 08 September 2021

\section{References}

1. Carlsson PI, Van Laer L, Borg E, Bondeson ML, Thys M, Fransen E, et al. The influence of genetic variation in oxidative stress genes on human noise susceptibility. Hear Res. 2005;202(1-2):87-96.

2. Sliwinska-Kowalska M, Pawelczyk M. Contribution of genetic factors to noise-induced hearing loss: a human studies review. Mutat Res. 2013;752(1):61-5.

3. Fridberger A, Flock A, Ulfendahl M, Flock B. Acoustic overstimulation increases outer hair cell $\mathrm{Ca}^{2+}$ concentrations and causes dynamic contractions of the hearing organ. Proc Natl Acad Sci USA 1998;95(12):7127-32.

4. Wang $X$, Zhu $Y$, Long $H$, Pan S, Xiong $H$, Fang $Q$, et al. Mitochondrial calcium transporters mediate sensitivity to noise-induced losses of hair cells and cochlear synapses. Front Mol Neurosci. 2018;11:469.

5. Orrenius S, Zhivotovsky B, Nicotera P. Regulation of cell death: the calcium-apoptosis link. Nat Rev Mol Cell Biol. 2003:4(7):552-65.

6. Yamane H, Nakai Y, Takayama M, Iguchi H, Nakagawa T, Kojima A. Appearance of free radicals in the guinea pig inner ear after noise-induced acoustic trauma. Eur Arch Oto-Rhino-Laryngol. 1995;252(8):504-8.

7. Yamashita D, Jiang HY, Schacht J, Miller JM. Delayed production of free radicals following noise exposure. Brain Res. 2004;1019(1-2):201-9.

8. Henderson D, Bielefeld EC, Harris $\mathrm{KC}, \mathrm{Hu} \mathrm{BH}$. The role of oxidative stress in noise-induced hearing loss. Ear Hear. 2006;27(1):1-19.

9. Robertson D. Functional significance of dendritic swelling after loud sounds in the guinea pig cochlea. Hear Res. 1983;9(3):263-78.

10. Abreu-Silva RS, Rincon D, Horimoto AR, Sguillar AP, Ricardo LA, Kimura L, et al. The search of a genetic basis for noise-induced hearing loss (NIHL). Ann Hum Biol. 2011;38(2):210-8.

11. Park SN, Back SA, Park KH, Seo JH, Noh HI, Akil O, et al. Comparison of functional and morphologic characteristics of mice models of noiseinduced hearing loss. Auris Nasus Larynx. 2013;40(1):11-7.

12. Michael AK, Fribourgh JL, Chelliah Y, Sandate CR, Hura GL, SchneidmanDuhovny $D$, et al. Formation of a repressive complex in the mammalian circadian clock is mediated by the secondary pocket of CRY1. Proc Natl Acad Sci USA. 2017;114(7):1560-5.

13. Rosensweig C, Reynolds KA, Gao P, Laothamatas I, Shan Y, Ranganathan $R$, et al. An evolutionary hotspot defines functional differences between cryptochromes. Nat Commun. 2018:9(1):1138.

14. Fontana JM, Tserga E, Sarlus H, Canlon B, Cederroth C. Impact of noise exposure on the circadian clock in the auditory system. J Acoust Soc Am. 2019;146(5):3960.

15. Takahashi JS. Transcriptional architecture of the mammalian circadian clock. Nat Rev Genet. 2017:18(3):164-79.

16. Basinou V, Park JS, Cederroth CR, Canlon B. Circadian regulation of auditory function. Hear Res. 2017;347:47-55.

17. Meltser I, Cederroth CR, Basinou V, Savelyev S, Lundkvist GS, Canlon B. TrkB-mediated protection against circadian sensitivity to noise trauma in the murine cochlea. Curr Biol. 2020;30(22):4547.

18. Park JS, Cederroth CR. Identification of a circadian clock in the inferior colliculus and its dysregulation by noise exposure. J Neurosci. 2016:36(20):5509-19.

19. Leake PA, Akil O, Lang $H$. Neurotrophin gene therapy to promote survival of spiral ganglion neurons after deafness. Hear Res. 2020;394:107955.

20. Hernández-Echeagaray E. Neurotrophin-3 modulates synaptic transmission. Vitam Horm. 2020;114:71-89.

21. Fariñas I, Jones KR, Tessarollo L, Vigers AJ, Huang E, Kirstein M, et al. Spatial shaping of cochlear innervation by temporally regulated neurotrophin expression. J Neurosci. 2001;21(16):6170-80.

22. Fritzsch B, Pirvola U, Ylikoski J. Making and breaking the innervation of the ear: neurotrophic support during ear development and its clinical implications. Cell Tissue Res. 1999;295(3):369-82.

23. Rubel EW, Fritzsch B. Auditory system development: primary auditory neurons and their targets. Annu Rev Neurosci. 2002;25:51-101. 
24. Yang T, Kersigo J, Jahan I, Pan N, Fritzsch B. The molecular basis of making spiral ganglion neurons and connecting them to hair cells of the organ of Corti. Hear Res. 2011;278(1-2):21-33.

25. Yang G, Yang Y, Tang H, Yang K. Loss of the clock gene Per1 promotes oral squamous cell carcinoma progression via the AKT/mTOR pathway. Cancer Sci. 2020;111(5):1542-54.

26. Zheng Y, Liu C, Li Y, Jiang H, Yang P, Tang J, et al. Loss-of-function mutations with circadian rhythm regulator Per1/Per2 lead to premature ovarian insufficiency. Biol Reprod. 2019;100(4):1066-72.

27. Shafi AA, McNair CM, McCann JJ, Alshalalfa M. The circadian cryptochrome CRY1, is a pro-tumorigenic factor that rhythmically modulates DNA repair. Nat Commun. 2021;12(1):401.

28. Lavebratt C, Sjöholm LK, Soronen P, Paunio T, Vawter MP, Bunney WE, et al. CRY2 is associated with depression. PLoS ONE. 2010;5(2):e9407.

29. Jiang R, Duan J, Windemuth A, Stephens JC, Judson R, Xu C. Genomewide evaluation of the public SNP databases. Pharmacogenomics. 2003;4(6):779-89.

30. Szobota S, Mathur PD, Siegel S, Black K, Saragovi HU, Foster AC. BDNF, NT-3 and Trk receptor agonist monoclonal antibodies promote neuron survival, neurite extension, and synapse restoration in rat cochlea ex vivo models relevant for hidden hearing loss. PLoS ONE. 2019;14(10):e0224022.

31. Green SH, Bailey E, Wang Q, Davis RL. The Trk A, B, C's of neurotrophins in the cochlea. Anat Rec. 2012;295(11):1877-95.

32. Fritzsch B, Tessarollo L, Coppola E, Reichardt LF. Neurotrophins in the ear: their roles in sensory neuron survival and fiber guidance. Prog Brain Res. 2004;146:265-78.

33. Chen S, Wang J, Cicek E, Roeder K, Yu H, Devlin B. De novo missense variants disrupting protein-protein interactions affect risk for autism through gene co-expression and protein networks in neuronal cell types. Mol Autism. 2020;11(1):76.

34. Chen S, Fragoza R, Klei L, Liu Y, Wang J, Roeder K, et al. An interactome perturbation framework prioritizes damaging missense mutations for developmental disorders. Nat Genet. 2018;50(7):1032-40.

35. Liu S, Liu L, Niu X, Lu D, Xia H, Yan S. A novel missense mutation (I26M) in DUOXA2 causing congenital goiter hypothyroidism impairs NADPH oxidase activity but not protein expression. J Clin Endocrinol Metab. 2015;100(4):1225-9.

36. Hoste C, Rigutto S, Van Vliet G, Miot F, De Deken X. Compound heterozygosity for a novel hemizygous missense mutation and a partial deletion affecting the catalytic core of the $\mathrm{H} 2 \mathrm{O} 2$-generating enzyme DUOX2 associated with transient congenital hypothyroidism. Hum Mutat. 2010;31(4):E1304-19.

37. Yonghong Zhou CD. General biology. Beijing: Higher Education Press; 2007. p. 125.

38. Miyagawa T, Hida A, Shimada M, Uehara C, Nishino Y, Kadotani H. A missense variant in PER2 is associated with delayed sleep-wake phase disorder in a Japanese population. J Hum Genet. 2019;64(12):1219-25.

39. Chu LW, Zhu Y, Yu K, Zheng T, Yu H, Zhang Y, et al. Variants in circadian genes and prostate cancer risk: a population-based study in China. Prostate Cancer Prostatic Dis. 2008;11(4):342-8.

40. Partch $\mathrm{CL}$, Green CB, Takahashi JS. Molecular architecture of the mammalian circadian clock. Trends Cell Biol. 2014;24(2):90-9.

41. Menet JS, Abruzzi KC, Desrochers J, Rodriguez J, Rosbash M. Dynamic PER repression mechanisms in the Drosophila circadian clock: from on-DNA to off-DNA. Genes Dev. 2010;24(4):358-67.

42. Koike N, Yoo SH, Huang HC, Kumar V, Lee C, Kim TK, et al. Transcriptional architecture and chromatin landscape of the core circadian clock in mammals. Science. 2012;338(6105):349-54.

43. Ye R, Selby CP, Chiou YY, Ozkan-Dagliyan I, Gaddameedhi S, Sancar A. Dual modes of CLOCK:BMAL1 inhibition mediated by cryptochrome and period proteins in the mammalian circadian clock. Genes Dev. 2014;28(18):1989-98.

44. Chiou YY, Yang Y, Rashid N, Ye R, Selby CP, Sancar A. Mammalian Period represses and de-represses transcription by displacing CLOCK-BMAL1 from promoters in a Cryptochrome-dependent manner. Proc Natl Acad Sci USA. 2016;113(41):E6072-9.

45. Yang $\mathrm{CH}$, Hwang $\mathrm{CF}$, Chuang $\mathrm{JH}$, Lian WS, Wang FS, Huang El, et al. Constant light dysregulates cochlear circadian clock and exacerbates noise-induced hearing loss. Int J Mol Sci. 2020;21(20):7535.

46. Yang CH, Hwang CF, Lin PM, Chuang JH, Hsu CM, Lin SF, et al. Sleep disturbance and altered expression of circadian clock genes in patients with sudden sensorineural hearing loss. Medicine. 2015;94(26):e978.
47. Mahmoudian-Sani MR, Mehri-Ghahfarrokhi A, Ahmadinejad F, Hashemzadeh-Chaleshtori M, Saidijam M, Jami MS. MicroRNAs: effective elements in ear-related diseases and hearing loss. Eur Arch Otorhinolaryngol. 2017;274(6):2373-80.

48. Zhao L, Wang J, Li Y, Song T, Wu Y, Fang S, et al. NONCODEV6: an updated database dedicated to long non-coding RNA annotation in both animals and plants. Nucleic Acids Res. 2021:49(D1):D165-71.

49. Chen J, Zhao X, Cui L, He G, Wang X, Wang F, et al. Genetic regulatory subnetworks and key regulating genes in rat hippocampus perturbed by prenatal malnutrition: implications for major brain disorders. Aging (Albany NY). 2020;12(9):8434-58

50. Zhou X, Li Q, Xu J, Zhang X, Zhang H, Xiang Y, et al. The aberrantly expressed miR-193b-3p contributes to preeclampsia through regulating transforming growth factor- $\beta$ signaling. Sci Rep. 2016;6:19910.

51. Jin G, Xu M, Zou M, Duan S. The processing, gene regulation, biological functions, and clinical relevance of N4-acetylcytidine on RNA: a systematic review. Mol Ther Nucleic Acids. 2020;20:13-24

52. Arango D, Sturgill D, Alhusaini N, Dillman AA, Sweet TJ, Hanson G, et al. Acetylation of cytidine in mRNA promotes translation efficiency. Cell. 2018:175(7):1872-86.e24.

53. Dominissini D, Rechavi G. N(4)-acetylation of cytidine in mRNA by NAT10 regulates stability and translation. Cell. 2018;175(7):1725-7.

54. Zhang Q, Liu H, Soukup GA, He DZ. Identifying microRNAs involved in aging of the lateral wall of the cochlear duct. PLOS ONE. 2014;9(11):e112857.

55. Zheng S, Zhao T, Yuan S, Yang L, Ding J, Cui L, et al. Immunodeficiency promotes adaptive alterations of host gut microbiome: an observational metagenomic study in mice. Front Microbiol. 2019:10:2415.

56. Wu Y, Cao H, Baranova A, Huang H, Li S, Cai L, et al. Multi-trait analysis for genome-wide association study of five psychiatric disorders. Transl Psychiatry. 2020;10(1):209.

57. Jiang L, Wang K, Lo K, Zhong Y, Yang A, Fang X, et al. Sex-Specific Association of circulating ferritin level and risk of type 2 diabetes: a doseresponse meta-analysis of prospective studies. J Clin Endocrinol Metab. 2019;104(10):4539-51.

58. Wang X, Wu W, Zheng W, Fang X, Chen L, Rink L, et al. Zinc supplementation improves glycemic control for diabetes prevention and management: a systematic review and meta-analysis of randomized controlled trials. Am J Clin Nutr. 2019;110(1):76-90.

59. Ji H, Dai D, Wang Y, Jiang D, Zhou X, Lin P, et al. Association of BDNF and BCHE with Alzheimer's disease: Meta-analysis based on 56 genetic case-control studies of 12,563 cases and 12,622 controls. Exp Ther Med. 2015;9(5):1831-40.

60. Xu M, Lin Z. Genetic influences of dopamine transport gene on alcohol dependence: a pooled analysis of 13 studies with 2483 cases and 1753 controls. Prog Neuropsychopharmacol Biol Psychiatry. 2011;35(5):1255-60.

61. Yu H, Pan R, Qi Y, Zheng Z, Li J, Li H, et al. LEPR hypomethylation is significantly associated with gastric cancer in males. Exp Mol Pathol. 2020;116:104493.

62. Liu M, Li F, Yan H, Wang K, Ma Y, Shen L, et al. A multi-model deep convolutional neural network for automatic hippocampus segmentation and classification in Alzheimer's disease. Neuroimage. 2020;208:116459.

63. Wang X, Fang X, Zheng W, Zhou J, Song Z, Xu M, Min J, Wang F. Genetic support of a causal relationship between iron status and type 2 diabetes: a Mendelian randomization study. J Clin Endocrinol Metab. 2021:dgab454. https://doi.org/10.1210/clinem/dgab454.

64. Zhang F, Baranova A, Zhou C, Cao H, Chen J, Zhang X, et al. Causal influences of neuroticism on mental health and cardiovascular disease. Hum Genet. 2021;140(9):1267-81.

65. Zhang F, Rao S, Cao H, Zhang X, Wang Q, Xu Y, Sun J, Wang C, Chen J, Xu X, Zhang N, Tian L, Yuan J, Wang G, Cai L, Xu M, Baranova A. Genetic evidence suggests posttraumatic stress disorder as a subtype of major depressive disorder. J Clin Invest. 2021:145942. https://doi.org/10.1172/JCl145942.

66. Hou L, Xu M, Yu Y, Sun X, Liu X, Liu L, et al. Exploring the causal pathway from ischemic stroke to atrial fibrillation: a network Mendelian randomization study. Mol Med. 2020;26(1):7.

\section{Publisher's Note}

Springer Nature remains neutral with regard to jurisdictional claims in published maps and institutional affiliations. 